\title{
The study of mnestic function and the attention of primary school children with learning difficulties
}

\author{
Vladislav Fadyeyev ${ }^{1 *}$, Mariya Fadyeyeva ${ }^{2}$ \\ ${ }^{1}$ V.I. Vernadsky Crimean Federal University, Taurida Academy, 295007, Simferopol, Russia \\ ${ }^{2}$ V.I. Vernadsky Crimean Federal University, Yevpatoriya institute of social sciences, \\ 297400, Yevpatoriya, Russia
}

\begin{abstract}
The article deals with the results of ascertaining experiment on research of mnestic function and the attention of primary school children with learning difficulties. There are two directions of correctional work with children with learning difficulties, the first of which embodies the idea of "replacement ontogenesis", the aim of the second approach is the development and correction of cognitive functions and their components.
\end{abstract}

The reasons of the learning difficulties of primary school children are variety. The difficulties in learning in the first year of study are often due to the pedagogical process itself: the overstrain of the content of training, the inflated level of requirements that are contained in the programs, the over-fullness of the class by primary school children, which does not allow for rational implementation of individual and differentiated approaches to learning. All this leads to such an overload, which without appropriate assistance from the outside, the primary school children cannot cope, because for this, in addition to abilities, it requires a lot of perseverance and efficiency. Difficulties in learning can also occur due to underdevelopment of the mnestic function of primary school children, as the inability of the child to remember affects his/her learning activities.

Memory is a collective concept that includes mnestic functions related to different modalities and categories. There is the following classification of that modalities and categories [1]:

1. Semantic memory refers to general factual knowledge and vocabulary, primarily associated with the left prefrontal and temporal divisions, including the limbic system. It is directly related to what the child learns in school. Semantically organized auditory information, which has a logical and abstract character, is more difficult to hold than visual information.

2. Episodic, or biographical, memory has to do with personally experienced events that have spatial and temporal boundaries. The left prefrontal and medial temporal divisions are mainly responsible for the storage of the relevant information, and the right prefrontal, posterior medial and medial temporal divisions are mainly responsible for the reproduction

\footnotetext{
* Corresponding author: vladislavfadeev@yandex.ru
} 
of the information. Strictly speaking, for the optimal functioning of episodic memory, it is necessary to realize the time and the use of ideas about time, which is possible not earlier than the age of four. Episodic and semantic memory are combined in the concept of descriptive memory (what can be explained). Descriptive memory contains information that characterizes "what", "where", "when", as well as "nice" or "unpleasant". However, these are different signs.

3. Procedural memory - non-declarative, or implicit, memory related to learning skills. It is associated mainly with the motor - frontal-parietal and subcortical (basal ganglia and cerebellum) areas of the brain. It stores information about how to do something, reading aloud, speech motor skills and possibly grammatical structure is also stored in procedural memory.

For an empirical study of the level of formation of mnestic functions and attention in primary schoolchildren with learning difficulties, we have identified a number of techniques that allow us to explore these features. These methods are:

1. The method of "Memorizing 10 words" by A. R. Luriya;

2. The method of "Retelling of the story" by A.V. Semenovich;

3 . The method "6 figures" by A.V. Semenovich;

4. The method "10 pairs of words" (L.F. Tikhomirova);

5. The method "Find 3-5 differences (how much time the child spends on finding) (A.V. Semenovich);

6. The Wexler's test (children's version. Subtest 7. Missing details) in the adaptation of Y. I. Filimonenko and V. I. Timofeev;

7. The Mann-Whitney's criterion.

In order to establish the specifics of the attention of younger students with learning difficulties, we investigated the features of observation and perceptual attention to identify the difficulties of programming and control of arbitrary forms of activity.

The results of the research of features of concentration and stability of attention of primary schoolchildren by a technique "Find 3-5 differences" (how many time the child spends on finding) (the author A. V. Semenovich) of the control and experimental groups are as follows: in the control group $86,67 \%$ of younger students found 5 differences, $13,37 \%$ found 4 differences. At the same time in the experimental group of 5 differences found $56,25 \%$ of primary school children, $25 \%$ of primary school students found 4 differences and $12,5 \%$ of primary school students with learning difficulties found 3 differences.

Analyzing the results of the study of the features of attention, observation of primary schoolchildren by the method of "Wexler Test (Children's version. Subtest 7. Missing parts) we have received the following data.

In the experimental group, $25 \%$ of the subjects were found a high level of perceptual attention volume (average of 17,5), which suggests that children were able to notice both the most important parts of the picture and small details. Children were able to see and analyze the entire object, to find the relationship of objects. In $43,75 \%$ - the average (average 15,28) - which means that children may not always notice the various elements of the object, and $31,25 \%$ - low (average 12,8), meaning that these children have a low level of observation, poor development of the ability to find meaningful components, and to conclude about their relationship.

In the control group of $56,25 \%$ of younger students showed a high level of scope of perceptual attention (secondary 17,66), 43,75\% - the average (mean of 15,42). There were no subjects with a low level of perceptual attention.

The calculation of the Mann-Whitney U-test $(\mathrm{p} \leq 0.05)$ showed that at the same speed of the task in the tests "Find 3-5 differences" and the test "Wexler (Children's version. Subtest 
7. Missing details)", successful learners are more attentive and make fewer mistakes than unsuccessful learners.

In order to establish the specifics of the mnestic function of younger students with learning difficulties, we studied the features of visual-perceptual, associative, short-term and operational memory to identify the difficulties of programming and control of arbitrary forms of activity.

During the examination of the state of auditory memory, a decrease in the volume and speed of direct and delayed verbal memorization in children of the experimental group compared with the control group was revealed. So, if children without difficulties in training on average memorized 10 words from the third time and after a break remembered 8 words, pupils with difficulties in training memorized words from the fourth time, at the delayed reproduction losing to 4-5 words. However, the structure of the memorization process was similar in all groups. It was characterized by a constant accumulation of memorized words with an increase in the number of presentations to $100 \%$ of reproduction ("growing curve"). It corresponded to the normative data for children of primary school age [1].

The edge factor, which consists mainly in naming the first or last words from the presented list, was practically not observed in any of the studied groups. At the same time, most of the children from the experimental group had the introduction of side wordsassociations. For example, when listing "cat-stump-..." the child continued, "the foresttrees...". But at repeated reproduction the reference number was called correctly, or was supplemented with new words. Such an introduction of side associations into mnestic activity observed in children with learning difficulties and easily corrected by them themselves with appropriate instructions may be associated with dysfunctional participation of the right hemisphere in mediating the processes of restructuring of perceived information [2].

According to the Mann-Whitney test, the differences between the control group and primary schoolchildren from the experimental group were statistically significant at $\leq 0.05$.

The general results of the study of random memory obtained by the method of "Retelling the story" (author AV Semenovich) younger students showed that in the control group of understanding, taking instructions, correctly completed the task revealed $71,4 \%$ of younger students, and asked for little help experimenter $28,6 \%$.

At the same time, in the experimental group, only $6,25 \%$ of younger students correctly completed the task, and with a little help, $25 \%$ of younger students of the experimental group coped. Most of the $56,25 \%$ partially completed the task, with the massive help of the experimenter. In addition, $13.5 \%$ of primary school children with learning difficulties with a massive help of only a few completed the task.

In the study of nonverbal memory by the method of "6 figures" a slight decrease in the volume and speed of direct and delayed memorization, although less pronounced than in verbal memorization, showed children with learning difficulties.

The children from the control group on average memorized six figures from 2 times and after the break played all the figures. The children from the experimental group for the full learning of a number of figures, it took up to 3 repetitions. When delayed playback after 2025 minutes without additional presentation of the standard, they remembered an average of 5 figures.

It is important to note that, although children with learning difficulties have shown some decrease in memory and memory strength, the exact image of the entire series from the third time in the presence of two errors or omissions of figures in delayed playback is, according to research data [2], within the norm for primary school age.

The most common errors of children with learning difficulties were structural-topological distortion: dysmetria, the distortion circuit, violations of the location of the parts of the figure. 
They were observed in $38,1 \%$ of the children from control group and $85,7 \%$ children of experimental group of cases, respectively.

The changes of the coordinate arrangement of elements, mirror, spatial errors occurred in $4.8 \%$ of children from the control group, in $47.6 \%$ of children from the experimental group. Errors of both types, characteristic of most children from the experimental group, indicate a lack of functional formation of the right hemisphere.

Many children with visual-spatial difficulties found ways to compensate them by "inventing" their mnemonics. For example, a child with learning difficulties described a difficult figure to remember as "similar to the number 4, the letter $M$ with a stick, a zigzag or step". Thus, he mediated the nonverbal figure with words, giving it a meaning that helped to remember it better.

The single errors in simplifying the configuration of figures and its incomplete reproduction were noted in 33,3\% children of control group and $61,9 \%$ children of experimental group. Children with learning difficulties demonstrate results on this parameter at the control group level. This fact suggests that the weakness of left-hemisphere information processing strategies, manifested in the errors of the described nature, is equally characteristic of children from the experimental and control groups.

The failure of the regulatory and dynamic components of mental activity is expressed in the repetition of the incorrect answer in subsequent playbacks. Perseveration of erroneous answers was manifested in $28,6 \%$ (control group), $67,1 \%$ (experimental group) of cases. That is, two-thirds of schoolchildren with learning difficulties had difficulties in active analysis and control of activities in the course of arbitrary memorization.

The described general features and differences of mnestic processes in the studied groups confirmed and illustrated by the following individual examples.

Example 1. Yuri K., 9 years, 3 class, right-handed. Control group.

With the tasks of verbal and non-verbal memorization, the child coped without difficulty. Thus, he listed all 10 words after three repetitions (7-9-10 words), not missing a single word in delayed playback. Sliding off the side of the association and of the factor of the region were noted. All six of the figures remembered in the correct order the second time. When delayed repetition of any element has not been lost, the order of reproduction was without violations. He reproduced 5-6-6 figures. In the first repetition there was one mistake additional detail of a contour which in the subsequent repetitions was corrected. Thus, scoolboy successfully completed the proposed tests, which confirms the formation of mnestic processes and brain mechanisms that ensure their implementation.

Example 2. Alex H., age 9, grade 3, right-handed. The experimental group.

Schoolchild took 3 repetition during the examination of verbal memory for a full memory (7-8-10). Under delayed playing, he mentioned 9 words. The first iterations of the met coined the words: "Keith, ray, honey, sleep, night, garden, ball, stump, ice, running" added "the cat, the mole, the hole, the juice". During the examination of nonverbal memory, the boy remembered all the figures in the correct order from the third time. In all repetitions there was a distortion of the figures. With delayed repetition, all elements were present, but their structure was not preserved. Overall, it was played 5-5-6-6 figures, including 10 (3-3-2-2) were performed with errors. The predominant number of errors occurred in connection with the violation of the structure of the reproduced figure - distortion of the contour of the figure, its simplification, loss of detail. There has been sporadic repetition of the error.

In general, the indicators of the volume and speed of memorization in both the auditory and visual spheres are within the limits of the norm. However, there are various distortions of memory traces, mainly non-verbal, presumably associated with right-hemisphere dysfunction.

Example 3. Anna T., 8 years old, 3rd grade, right-handed. The experimental group. 
The girl remembers all of the words after 5 repetitions. With delayed playback called 8 words. There was frequent slippage on the side associations, which changed with each new repetition ("glasses, mushroom, juice"). It took two repetitions to memorize all the pieces in the correct order. However, the distortion of the figures noted in the third and in the fourth repetition. Under the prolonged repetition of a single element was not lost. Overall, it was played 5-6-6-6 figures, of which 15 (4-4-4-3) were performed with errors. Most of the distortion was wearing structural-topological nature - the distortion of the shape's outline. There were some simplifications, the transformation of the figure into a sign.

The child demonstrated a lower level of development of memory processes: reduced speed of memorization and the amount of memorized material, there are more errors. This shows the insufficient degree of formation of the functions of reception, processing and storage of information, and providing their brain mechanisms.

Neuropsychological study of memory processes revealed qualitative and quantitative differences in the mnestic sphere in the study groups. At the same time, the revealed memory disorders in the auditory and visual modalities were not homogeneous. The difficulties of verbal memory in its severity by both qualitative and quantitative aspects in children with learning difficulties wore structurally unstable in nature and expressed primarily in pseudocopulation, tumors disappearing when the subsequent iteration. At the same time, in visual memory, the violation of the order of reproduction was combined with an abundance of distortions and transformations of the reference figures. The presented images were modified in connection with various reversals, metric and structural contamination. These phenomena reflect the state of visual and visual-spatial functions, and, consequently, are provided by the activity of the posterior parts of the right hemisphere, the dysfunction of which entails the unformed spatial and simultaneous factors, expressed in the described errors.

The children with learning difficulties have a decrease in the volume and speed of attention and memorization of verbal and non-verbal information. The average number of spatial and regulatory errors and the percentage of children who performed the task with different errors are increasing. Differences in the amount of attention, memorization and reproduction of verbal stimuli to a certain extent depend on the processes of arbitrary regulation and control, when there is a strong intention to remember words and actively remember them after a time interval. The development of these functions is carried out by the structures of the front departments of the left hemisphere.

Thus, we can distinguish 6 children of the "risk group" (Anna T., Alexander M., Pavel S., Nikita S., Alexey H. and Valentin K.) according the results of all the children of the experimental group. They have the most pronounced learning difficulties, mainly due to insufficient formation of programming and control of arbitrary forms of activity.

The difficulties of these children were manifested in the fact that they could not as quickly as the other children to understand the task and follow the instructions until the end of its implementation. They characterized by incomplete orientation in the conditions of the task, rash impulsive answers, and frequent lack of verification of the result with a given sample. In the behavior of one child (Anna T.) there was a decrease in the dynamics of attention, increased distraction, her actions were often inconsistent and chaotic. The other 5 children, on the contrary, were characterized by slowness, lethargy, inactivity, expressed to a greater or lesser extent, as well as insufficient interest in the tasks performed.

Neuropsychological research has shown that the difficulties of programming and control of arbitrary forms of activity combined in some children with some underdevelopment and other higher mental functions. If Anna T. had difficulties in programming and control on the background of hyperactivity were relatively isolated, Valentine K. they accompanied by 
increased depletion, fluctuations in performance due to the lack of the energy block of the brain.

Unlike Anne T. and Valentin, K. the remaining 4 children (Alexander M., Paul S., Nikita S., Alex H.) in addition to the difficulties of programming and control was also present issues associated with the receiving unit and information processing. There was a slight underdevelopment of phonemic analysis and verbal memory, and three of them - the difficulty of the spatial character and the weakness of visual perception and memory. These children with learning difficulties need to justify the directions and content of recommendations for the development of mnestic function and attention.

There are two main areas of correctional work.

In the first direction, proposed by A. V. Semenovich, the idea of "replacement ontogenesis" is embodied. Such corrective techniques are aimed at the formation of the basic foundations, prerequisites of cognitive functions, and proceed from the fact that the impact on the sensorimotor level activates the development of all higher mental functions. They use a hierarchical complex of motor (body-oriented) methods and cognitive methods, taking into account their complementary effects [3].

The second direction, actively developed in the laboratory of the study of learning difficulties under the guidance of T. V. Akhutina, aimed to the development and correction of cognitive functions and their components [4].

So, T. V. Akhutina and N. M. Pylaeva prepared two clear system programs to overcome the difficulties of the doctrine associated with the insufficient development of the functions of the second and third blocks of the brain.

The first program includes a methodical complex of tasks for the development of visualverbal and visual-spatial functions.

The second program ("School of attention") - methods of development and correction of programming and control functions.

\section{References}

1. R. Cabeza R. \& L. Nyberg Neural bases of learning and memory: Functional neuroimaging evidence (2000)

2. L.S. Tsvetkova, The actual problems of neuropsychology of the childhood: textbook ("MODEC", Moscow, 2001)

3. A.V. Semenovich, The introduction to the neuropsychology of the childhood (Genesis, 2012)

4. T.V. Akhtunina, The overcome the difficulties of the teaching: neuropsychological approach (Piter, 2008) 